\title{
Estudo da adaptação da espécie Piper hispidinervum C. DC. (pimenta longa) à região do Vale do Itajaí - SC, através da composição química do óleo essencial obtido por hidrodestilação por micro-ondas e convencional
}

\author{
Dilamara RIVA ${ }^{1}$, Edésio Luiz SIMIONATTO², Alberto WISNIEWSKI JR ${ }^{3}$, Airton Rodrigues SALERNO ${ }^{4}$, \\ Terezinha Heck SCHALLENBERGER ${ }^{5}$
}

\section{RESUMO}

Neste trabalho, a espécie Piper hispidinervum (pimenta longa), endêmica do estado do Acre, foi cultivada na regiāo do Vale do Itajaí - SC, e sua adaptação foi avaliada em relação à composição química do óleo essencial obtido pelos processos de hidrodestilação convencional e por micro-ondas. O safrol foi identificado como o constituinte majoritário do óleo essencial desta espécie, o qual foi empregado como parâmetro de avaliação da capacidade de adaptação desta planta à região sul do Brasil, já que a proposta abrange avaliar a utilização desta espécie como fonte alternativa de safrol, em substituição a Canela Sassafrás (Ocotea odorifera), espécie muito explorada até a década de 90 nesta regiāo. As amostras da planta foram obtidas de diferentes regióes do Acre e foram cultivadas na estação experimental da EPAGRI - Itajaí-SC. O óleo essencial das folhas forneceu um teor médio de safrol entre 76,6\% e 89,9\%. A análise por CG-DIC e CG-EM do óleo com maior concentração de safrol, apresentou os seguintes constituintes: safrol (89,93\%), $\alpha$-terpineno $(0,35 \%)$, (E)- $\beta$-ocimeno $(0,54 \%)$, terpinoleno $(3,10 \%)$, valenceno $(0,21 \%),(Z)$ - $\beta$-bisaboleno $(1,70 \%)$ e guaiol $(0,29 \%)$.

PALAVRAS-CHAVE: óleo essencial, pimenta longa, safrol, micro-ondas, Piper hispidinervum.

\section{Adaptation studies of Piper hispidinervum C. DC. (long pepper) species in Itajaí Valley - SC, by the chemical composition of essential oil obtained by microwave and traditional hydrodistillation}

\begin{abstract}
In this work a Piper hispidinervum specie (long pepper), endemic from the Acre state, was grown in Itajaí Valley in Santa Catarina State, and its adaptation was evaluated in relation to chemical composition of essential oil obtained by traditional hydrodistillation and microwave hydrodistillation. The safrole was identified as the major constituent of the essential oil of this specie, which was used as a measure of evaluation of the adaptation capacity of this plant in the southern Brazil, as the proposal includes the evaluation of the usage of this specie as an alternative source of safrole, replacing Sassafras Cinnamon (Ocotea odorifera), widely explored specie in this region until the 90's decade. The plant samples were obtained from different regions of the Acre state and were cultivated at the experimental station of EPAGRI Itajaí - SC. The essential oil of the leaves provided an average content of safrole between $76.6 \%$ and $89.9 \%$. The analysis by GC-FID and GC-MS of the oil with higher safrole content, presented the following relative constitution: safrole $(89.93 \%)$, $\alpha$-terpinene $(0.35 \%)$, (E)- $\beta$-ocimene $(0.54 \%)$, terpinolene (3.10\%), valencene (0.21\%), (Z)- $\beta$-bisabolene $(1.70 \%)$ and guaiol $(0.29 \%)$.
\end{abstract}

KEYWORDS: essential oil, long pepper, safrole, microwave, Piper hispidinervum.

\footnotetext{
1 Universidade Regional de Blumenau. dilaquimica@hotmail.com

2 Universidade Regional de Blumenau. edesio@furb.br

3 Universidade Federal de Sergipe, albertowj@ufs.br

${ }^{4}$ EPAGRI - Itajaí - SC. salerno@epagri.sc.gov.br

${ }^{5}$ EPAGRI - Itajaí - SC. tcheck@epagri.sc.gov.br
} 


\section{INTRODUÇÃO}

A demanda mundial por óleos ricos em safrol é bastante expressiva. $\mathrm{O}$ valor industrial do safrol decorre da presença do grupo piperonila em sua molécula, a partir do qual, com pequenas transformaçóes químicas, obtêm-se compostos orgânicos com vasto emprego comercial, como é o caso do piperonal, usado na indústria de fragrâncias e perfumes finos e do butóxido de piperonila, agente sinergístico do piretro, inseticida natural, com certificação "verde e biodegradável", sem os riscos dos inseticidas sintéticos (Braga et al. 2005; Bandoni e Czepak 2008; Maia e Andrade 2009).

O Brasil já foi o maior produtor mundial de safrol, tendo no Vale do Itajaí (SC) a maior região produtora através da extração de Canela Sassafrás (Ocotea odorifera). Com a proibição do corte da Canela Sassafrás em 1991, pelo IBAMA, em função do risco de extinção da planta, o Brasil passou de exportador, a importador do produto (Valle 2003).

A eventual redução na oferta de safrol é uma das ameaças para a indústria de inseticidas piretróides e a descoberta de fontes sustentáveis para a produção desta matéria-prima é extremamente relevante, principalmente considerando-se a tendência de aumento de consumo de butóxido de piperonila (Bizzo et al. 2009).

Piper hispidinervum (pimenta longa) é um arbusto endêmico do estado do Acre com alto rendimento em óleo essencial rico em safrol (em torno de 90\%), descoberta na década de 70, por técnicos do Instituto Nacional de Pesquisas da Amazônia (INPA) (Aguiar 2003). É uma planta rústica, muito exigente em água e luz e aparece com frequência em áreas de capoeira (Embrapa 2000). Esta espécie pode ser propagada tanto por sementes, quanto por estacas e alcança de 1,60 a 1,80 m de altura e um ciclo vegetativo rápido de aproximadamente 1 a 2 anos (Maia et al. 1987).

O primeiro trabalho de caracterização da espécie Piper hispidinervum foi realizado por Gottlieb et al. (1981), onde o teor de safrol relatado foi de $89 \%$. A partir deste, muitos estudos foram realizados com esta espécie, com variaçōes no teor de safrol. Segundo Silva e Oliveira (2000), o teor de safrol variou de $87 \%$ a $97 \%$, no cultivo de pimenta longa no estado do Acre. Miranda (2002) avaliou o teor de safrol em plantas nativas da cidade de Xapuri - AC e observou um teor superior a 92\%. Já, Andrade et al. (2006) obtiveram rendimento de safrol de $72,3 \%$, em plantas coletadas em áreas próximas à cidade de Rio $\mathrm{Branco}-\mathrm{AC}$ e propagadas por semente na cidade de Belém - PA. O teor de safrol de $82,5 \%$ foi encontrado em pesquisa realizada por Nascimento et al. (2008), em plantas cultivadas na cidade de Lavras - MG. Pesquisas de Zacaroni et al. (2009), mostraram que o óleo essencial extraído de pimenta longa também cultivada em Lavras - MG apresentou um rendimento de safrol de $89 \%$.
Hoje, os únicos produtores mundiais de safrol são China e Vietnã que usam os mesmos métodos destrutivos que eram empregados no Brasil na época da Canela Sassafrás, em Santa Catarina. Tal prática compromete a oferta de safrol natural a longo prazo (Embrapa 2000).

Neste contexto, a pimenta longa torna-se uma fonte alternativa de safrol natural que pode ser explorada de forma náo destrutiva, pois o óleo essencial encontra-se nas folhas da planta que rebrota com facilidade após o corte (Embrapa 2000).

No processo de extração de óleos essenciais podem ser aplicados diversos métodos. Hidrodestilaçáo com aquecimento por microondas é uma técnica que tem como principal vantagem a redução do tempo de extração. Pode-se atribuir isto ao fato de que a irradiação por micro-ondas produz mais rapidamente calor no interior da glândula oleífera, bem como no seu exterior (Lucchesi et al. 2004; Bandoni e Czepak 2008; Golmakani e Rezaei 2008).

$\mathrm{Na}$ tentativa de reativar a indústria extrativa de safrol no estado de Santa Catarina, o presente trabalho teve por objetivo avaliar a melhor matriz das plantas da espécie Piper hispidinervum, de diferentes localizaçôes provenientes do estado do Acre, cultivadas na Estação Experimental da EPAGRI - SC. Bem como, comparar a hidrodestilaçáo com aquecimento convencional com a hidrodestilação com aquecimento por micro-ondas e caracterizar quimicamente o óleo essencial desta espécie. O safrol, constituinte majoritário, foi utilizado como parâmetro de avaliação da capacidade de adaptação desta planta à regiáo sul do Brasil.

\section{MATERIAL E MÉTODOS}

O material propagativo foi coletado de regióes distintas nas proximidades da cidade de Rio Branco no ano de 1995 e cultivado na Estação Experimental da EPAGRI em Itajaí SC, localizada a uma latitude de 260 57' 57" sul e longitude $48^{\circ} 48^{\prime} 01^{\prime \prime}$ oeste. A coleção cultivada na EPAGRI, apresenta 6 exemplares. Para diferenciar foi atribuído as identificaçōes: 02, 03, 04 e 06, sendo que os exemplares 01 e 05 foram perdidos (Tabela 1). Em outubro de 2008 foram coletadas folhas, das localizaçóes 02, 03, 04 e 06, divididas por planta e subdivididas por altura, sendo "A" folhas de meia altura até o topo e "B" da base até meia altura. Em abril de 2009 foi coletado material vegetal apenas da localização 03 , para estudo comparativo entre extração com aquecimento convencional e por micro-ondas. A secagem do material vegetal foi feita a temperatura ambiente por um período de 8 dias (Pimentel et al. 1998). Esta espécie foi identificada pelo botânico André Luís de Gasper e a exsicata da planta encontra-se depositada no Herbário Dr. Roberto Miguel Klein - FURB (Universidade Regional de Blumenau) sob o registro 9997. 
Tabela 1 - Procedência do material propagativo.

\begin{tabular}{lc}
\hline Identificação & Localização \\
\hline 01 & BR 364, Rio Branco - Porto Velho, Ramal km 18 (perdida) \\
02 & BR 364, Rio Branco - S. Madureira, Vila Custódio \\
03 & BR 364, Rio Branco - S. Madureira, km 22 \\
04 & Bujarí, Ramal do Cemitério, encosta \\
05 & Bujarí, Ramal do Cemitério, baixada (perdida) \\
06 & Bujarí, Ramal 2 \\
\hline
\end{tabular}

O material vegetal seco e triturado (30 gramas) foi submetido à hidrodestilaçáo convencional, em aparelho de Clevenger modificado (Gottlieb e Magalhāes 1960), durante 4 horas (Santos et al. 2004). Para realização da extração do óleo essencial através do aquecimento por micro-ondas foi utilizado um forno tipo doméstico, marca Philco, modelo PMW-101, com potência de $800 \mathrm{~W}$ e frequência de micro-ondas de 2450 MHz. Fez-se necessário retirar o prato interno, eliminando assim o sistema de rotaçáo. Na parte superior do forno fora feito um orifício, a fim de permitir a ligaçấo do balão ao condensador. Uma coluna de adaptação foi colocada entre a boca do baláo e o aparelho de Clevenger, a este, conectou-se um condensador Allihn com 5 bolas, precedido da conexão ao sistema de refrigeração em banho termostatizado a uma temperatura de $9^{\circ} \mathrm{C}$. O tempo de extraçáo foi de 25 minutos. A fase aquosa foi extraída com diclorometano $\left(\mathrm{CH}_{2} \mathrm{Cl}_{2}\right)$, secou-se a fase orgânica com sulfato de sódio anidro $\left(\mathrm{Na}_{2} \mathrm{SO}_{4}\right)$ e concentrou-se a pressão reduzida em evaporador rotatório. O rendimento de óleo essencial foi determinado em relaçáo à massa seca. Os extratos foram acondicionados em frasco apropriado e armazenados em refrigerador para posterior análise.

A quantificação absoluta de safrol foi determinada em Cromatógrafo Líquido de Alta Eficiência (CLAE), Modelo VARIAN PROSTAR 230/310, detector de UV $(\lambda=287 \mathrm{~nm})$; coluna $\mathrm{C}_{8}$ fase reversa $(250 \times 4,6 \mathrm{~mm})$, tamanho de partícula de $5 \mu \mathrm{m}$; injeçáo manual, volume injetado de $20 \mu \mathrm{L}$, sendo a fase móvel constituída de metanol (50\%), água (35\%) e acetonitrila (15\%). Prepararam-se soluçôes com padrão de safrol (ALDRICH) nas concentraçóes de 10, 25, 50, 100 e $200 \mu \mathrm{g} \cdot \mathrm{mL}^{-1}$. As amostras foram diluídas em metanol na concentração de $100 \mu \mathrm{g} \cdot \mathrm{mL}^{-1}$ (Archer 1988).

A caracterização química realizada através de CG-DIC, modelo SHIMADZU $14 \mathrm{~B}$, coluna OV-5 (30 m x 0,25 mm d.i. $\mathrm{x} 0,25 \mu \mathrm{m}$ de espessura do filme). Foi utilizado $\mathrm{N}_{2}$ como gás de arraste, a uma pressão constante de $80 \mathrm{kPa}$. A razão do split foi de $1 / 150$ e foi injetado $0,2 \mu \mathrm{L}$ do óleo puro. A temperatura do detector foi de $300{ }^{\circ} \mathrm{C}$ e do injetor de 250 ${ }^{\circ} \mathrm{C}$. A temperatura inicial da coluna foi de $50{ }^{\circ} \mathrm{C}(3 \mathrm{~min})$, aumento de $5^{\circ} \mathrm{C} \mathrm{min}^{-1}$ até $270{ }^{\circ} \mathrm{C}(8 \mathrm{~min})$, resultando em um tempo total de análise de 55 minutos (Adams 2007). Do óleo com maior rendimento de safrol fez-se a análise por
CG-EM, modelo VARIAN CP 3800, com ionização por elétrons de $70 \mathrm{eV}$ e temperatura da fonte de íons de $170{ }^{\circ} \mathrm{C}$. A composição química do óleo e a quantificação relativa foram determinadas por CG-DIC, através do índice de retenção (IR) dos compostos em relação a uma série homóloga de n-alcanos e confirmados por CG-EM através da comparação do espectro de massas dos compostos com a espectroteca NIST 02. Para avaliar a exatidáo dos valores de IR calculados para os componentes voláteis determinou-se os IR de padrôes secundários (heptanal, limoneno, guaiacol, cânfora, transanetol, safrol, timol, eugenol e tercbutilhidroxitolueno ) submetidos às mesmas condiçôes de análise.

\section{RESULTADOS E DISCUSSÃO}

A espécie Piper hispidinervum apresentou um teor de umidade de $73,90 \% \pm 2,14$. Constatou-se que o rendimento de óleo essencial, em relação à massa seca de pimenta longa variou entre $2,94 \%$ e 5,42\%. As extraçóes feitas com folhas de meia altura até o topo da planta, forneceram um rendimento de óleo essencial maior em relação às folhas da base até meia altura (Tabela 2). Gobbo-Neto e Lopes (2007), atribuem isso ao fato de que tecidos mais novos geralmente possuem maior taxa biossintética de metabólitos, tais como óleos essenciais.

Foi observada uma variação no teor absoluto de safrol entre 76,6\% e 89,9\%, no óleo essencial de Piper hispidinervum cultivada no Vale do Itajaí - SC. Nascimento et al. (2008) e Zacaroni et al. (2009), constataram um teor de safrol de 82,5\% e $89 \%$ respectivamente, para a planta em sua regiáo endêmica.

Tabela 2 - Rendimento de óleo essencial em folhas secas de Piper hispidinervum cultivada em Itajaí - SC, e teor de safrol no óleo essencial obtido.

\begin{tabular}{lcc}
\hline Amostra & Rendimento óleo $(\%)(\mathrm{m} / \mathrm{m})$ & Teor safrol (\%) (CLAE-UV) \\
\hline H1A02 & 4,38 & 89,9 \\
H1B02 & 4,35 & 81,3 \\
H1A03 & 5,42 & 81,9 \\
H1B03 & 4,33 & 82,9 \\
H1B03f+g & 3,68 & 85,2 \\
H1A04 & 5,18 & 76,9 \\
H1B04 & 3,81 & 76,6 \\
H1A06 & 3,68 & 78,4 \\
H1B06 & 2,94 & 77,1 \\
\hline
\end{tabular}

$A=$ folhas de meia altura até 0 topo; $B=$ folhas da base até meia altura; $f+g=$ folhas + galhos; $\mathrm{H} 1$ = hidrodestilação; 02 a 06 = diferentes procedências da espécie.

As melhores matrizes para propagação desta espécie foram das localizaçóes 02 e 03 , as quais apresentaram um maior teor de safrol. Uma vez que não houve diferenças no cultivo das mudas na Estação Experimental em SC, a variação nos rendimentos pode ser atribuída à procedência das plantas. Cada planta da coleção cultivada na EPAGRI - SC foi obtida de regióes distintas das proximidades de Rio Branco, no estado do Acre. 
Com o material da localização 03 , foram feitos experimentos utilizando-se folhas e também folhas + galhos. Foi observado que os rendimentos de óleo foram superiores nos experimentos utilizando-se somente folhas $(A=5,42 \%$; $\mathrm{B}=4,33 \%)$ do que no experimento com folhas + galhos $(3,68 \%)$, porém o maior índice do teor absoluto de safrol foi obtido na amostra com folhas + galhos $(85,2 \%)$. O teor absoluto de safrol foi determinado empregando-se uma curva de calibração obtida através da análise de soluçóes de um padrão primário de safrol.

Foi identificada $96,12 \%$ da composição química relativa do óleo, sendo que dos sete compostos majoritários identificados, aproximadamente $90 \%$ corresponde apenas ao safrol, um fenilpropanóide; os demais se caracterizam por serem monoterpenos: $\alpha$-terpineno (0,35\%), (E)- $\beta$-ocimeno $(0,54 \%)$, terpinoleno $(3,10 \%)$ e sesquiterpenos: valenceno $(0,21 \%)$, (Z)- $\beta$-bisaboleno $(1,70 \%)$ e guaiol $(0,29 \%)$. Os constituintes majoritários, segundo Andrade et al. (2006), em estudo do óleo essencial de $P$. hispidinervum cultivada na cidade de Belém - PA, foram: canfeno $(0,2 \%), \delta$-3-careno $(1,4 \%)$, (E)- $\beta$-ocimeno $(2,0 \%)$, terpinoleno $(10,5 \%)$, isoborneol $(0,1 \%)$, safrol $(72,3 \%)$, miristina $(2,4 \%)$, guaiol $(0,5 \%), \beta$-eudesmol $(0,1 \%)$ e bulnesol $(0,2 \%)$. Se comparados os constituintes dos dois óleos essenciais provenientes de regiôes diferentes, percebe-se que alguns componentes são idênticos e além do safrol o outro constituinte obtido em maior porcentagem é o terpinoleno.

A Tabela 3 apresenta os constituintes principais das folhas secas de P. hispidinervum cultivada no Vale do Itajaí - SC.

Foi observada uma diferença no índice de retenção calculado do safrol (1327), comparado ao da literatura (1285) (Adams 2007). Isto se deve ao fato de que a concentraçâo de safrol neste óleo é bastante elevada, o que causa um alargamento da base do pico, fornecendo um valor do tempo de retenção equivocado. Quando o óleo essencial foi diluído e injetado no CG-DIC forneceu um índice de retenção de 1293, próximo ao da literatura.

Tabela 3 - Constituintes principais do óleo essencial de $P$. hispidinervum cultivada no sul do Brasil referente a amostra H1A03 (CG-DIC).

\begin{tabular}{lccc}
\hline Constituinte & IR Literatura & IR Calculado & \% Relativa (CG-DIC) \\
\hline$\alpha$-Terpineno & 1014 & 1017 & 0,35 \\
(E)- $\beta$-Ocimeno & 1044 & 1054 & 0,54 \\
Terpinoleno & 1086 & 1095 & 3,10 \\
Safrol & 1285 & 1327 & 89,93 \\
Valenceno & 1496 & 1487 & 0,21 \\
(Z)- $\alpha$-Bisaboleno & 1506 & 1505 & 1,70 \\
Guaiol & 1600 & 1600 & 0,29 \\
& & Total & 96,12 \\
\hline
\end{tabular}

$\mathrm{Na}$ coleta do material vegetal realizada em abril de 2009, foram realizadas extraçóes dos óleos essenciais de pimenta longa por duas técnicas diferentes: hidrodestilação com aquecimento convencional (H3A03-HC) e hidrodestilação com aquecimento por micro-ondas (H3A03-M1). O óleo essencial obtido por extração em micro-ondas forneceu aumento tanto no rendimento de óleo, quanto no teor absoluto de safrol (Tabela 4). Golmakani e Rezaei (2008) atribuem isto ao fato de que a irradiaçáo de micro-ondas

Tabela 4 - Rendimento do óleo essencial de Piper hispidinervum e teor absoluto de safrol por diferentes métodos extrativos (Coleta abril/2009).

\begin{tabular}{lcc}
\hline Amostra & Rendimento óleo (\%) & Teor safrol (\%) (CLAE-UV) \\
\hline H3A03-HC & 3,56 & 77,8 \\
H3A03-M1 & 4,32 & 86,1 \\
\hline
\end{tabular}

$A=$ folhas de meia altura até 0 topo; $H 3$ = hidrodestilação; 03 = procedência da espécie; $\mathrm{HC}=$ hidrodestilação convencional; $\mathrm{M} 1$ = hidrodestilação por microondas.

causa ruptura na parede glandular da folha que se desintegra mais rápida e eficientemente.

Uma das maiores vantagens de se utilizar a técnica de extração por micro-ondas é a redução do tempo de extração. A extração por micro-ondas foi realizada em 25 minutos, enquanto que a extraçáo por aquecimento convencional teve duração de 240 minutos.

Foi verificado que após 25 minutos ambos os processos atingem a estabilidade no percentual de óleo obtido, destacando o processo por micro-ondas, o qual otimiza a recuperaçáo do produto com um aumento de $21,35 \%$.

\section{CONCLUSÕES}

$\mathrm{O}$ uso da energia de micro-ondas na indústria extrativa do óleo essencial de pimenta longa pode ser uma alternativa para reduzir o tempo de extração e aumentar o rendimento de óleo.

Com estes resultados, mostrou-se que a pimenta longa apresentou boa adaptação no Vale do Itajaí - SC, tendo rendimento de óleo e teor de safrol próximos aos obtidos em cultivos em outras regióes do Brasil, podendo assim ser uma alternativa para a produção de safrol na regiâo sul do Brasil e uma possibilidade atraente para a indústria agroindustrial extrativa de SC.

\section{AGRADECIMENTOS}

À FAPESC; CAPES-PROSUP; EPAGRI - Itajaí e FURB (Universidade Regional de Blumenau). 


\section{BIBLIOGRAFIA CITADA}

Adams, R.P. 2007. Identification of essential oil components by gas chromatography, mass spectroscopy. 4 ed., Carol Stream: Allured. 804 pp.

Andrade, E.H.A.; Guimarães, E.F.; Silva, M.H.L.; Maia, J.G.S. 2006. O óleo essencial de Piper hispidinervum C. DC. proveniente de propagação por semente. Resumo da 29a Reunião Anual da Sociedade Brasileira de Química, Águas de Lindóia, São Paulo.

Aguiar, E.M. 2003. Isolamento e caracterizaçâo de óleos essenciais de Piperáceas no Vale do Itajaí, Santa Catarina. Dissertação de Mestrado, Universidade Federal de Santa Catarina, Florianópolis, Santa Catarina. 106 pp.

Archer, A.W. 1988. Determination of safrole and myristicin in nutmeg and mace by high-performance liquid chromatography. Journal of Chromatography, 438: 117-121.

Bandoni, A.L.; Czepak, M.P. 2008. Os recursos vegetais aromáticos no Brasil: seu aproveitamento industrial para a produção de aromas e sabores. EDUFES, Vitória, ES. 623 pp.

Bizzo, H.R.; Hovell, A.M. C.; Rezende, C.M. 2009. Óleos essenciais no Brasil: aspectos gerais, desenvolvimento e perspectivas. Quimica Nova, 32(3): 588-594.

Braga, N.P.; Cremasco, M.A.; Valle, R.C.C.R. 2005. The effects of fixed-bed drying on the yeld and composition of essential oil from long pepper (Piper hispidinervum C. DC.) leaves. Brazilian Journal of Chemical Engineering, 22: 257-262.

EMBRAPA, 2000. Centro de informação de Pimenta Longa. (http:// www22.sede.embrapa.br/pimentalonga/). Acesso em 05/02/09.

Gobbo-Neto, L.; Lopes, N.P. 2007. Plantas medicinais: fatores de influência no conteúdo de metabólitos secundários. Química Nova, 30 (2): 374-381.

Golmakani, M.T.; Rezaei, K. 2008. Comparison of microwaveassisted hydrodistillation with the traditional hydrodidtillation method in the extraction of essential oils from Thymus vulgaris L. Food Chemistry, 109: 925-930.

Gottlieb, O.R.; Koketsu, M.; Magalhaes, M.T.; Maia, J.G.S.; Mendes, P.H.; Rocha, A.I.; Silva, M.L.; Wilberg, V.C. 1981. Óleos essenciais da Amazônia. Acta Amazonica, 11: 143-148.

Gottlieb, O.R.; Magalhães, M.T. 1960. Modified distillation trap. Chemist Analyst, 49: 114-116.
Lucchesi, M.E.; Chemat, F.; Smadja, J. Solvente-free microwave extraction of essential oil from aromatic herbs: comparison with conventional hydro-distillation. 2004. Jornal of Chromatography A, 1043: 323-327.

Maia, J.G.S.; Silva, M.L.; Luz, A.I.R.; Zoghbi, M.G.B.; Ramos, L.S. 1987. Espécies de Piper da Amazônia ricas em safrol. Química Nova, 10: 200-204.

Maia, J.G.S.; Andrade, E.H. 2009. Database of the amazon aromatic plants and their essential oils. Quimica Nova, 32(3): 595-622.

Miranda, E.M. 2002. Caracterização e avaliação produtiva de uma população nativa de Pimenta Longa (Piper hispidinervum) no Seringal Cachoeira, AC, Brasil. Acta Amazonica, 32(1): 9-20.

Nascimento, F.R.; Cardoso, M.G.; Souza, P.E.; Lima, R.K.; Salgado, A.P.S.P.; Guimarães, L.G.L. 2008. Efeito do óleo essencial de pimenta longa (Piper hispidinervum C. DC.) e do emulsificante Tween ${ }^{\circledR} 80$ sobre o crescimento micelial de Alternaria alternata (Fungi: Hyphomycetes). Acta Amazonica, 38(3): 503-508.

Pimentel, F.A.; Cabral, W.G.; Silva, M.R.; Pinheiro, P.S.N. 1998. Processo de secagem de biomassa de pimenta longa (Piper ispidinervum). Comunicado Técnico, 98. Embrapa Acre.

Santos, A.S.; Alves, S.M.; Figueiredo, F.J.C.; Neto, O.G.R. 2004. Descrição de sistema e de métodos de extração de óleos essenciais e determinação de umidade de biomassa em laboratório. Comunicado Técnico, 99. Embrapa PA, Belém, Pará.

Silva, A.C.P.R.; Oliveira, M.N. 2000. Caracterização botânica e química de três espécies do gênero Piper no Acre. Boletim de Pesquisa 23. Embrapa AC, Rio Branco, Acre.

Valle, R.C.C.R. 2003. Estratégias de cultivo de células de pimenta longa (Piper hispidinervum) e determinação de parâmetros cinéticos. Tese de Doutorado, Universidade Federal de Santa Catarina, Florianópolis, Santa Catarina. 184 pp.

Zacaroni, L.M.; Cardoso, M.G.; Souza, P.E.; Pimentel, F.A.; Guimaraes, G.L.; Salgado, A.P.S.P. 2009. Potencial fungitóxico do óleo essencial de Piper hispidinervum (pimenta longa) sobre os fungos fitopatogênicos Bipolaris sorokiniana, Fusarium oxysporum e Colletotrichum gloeosporioides. Acta Amazonica, 39(1): 193-198.

Recebido em 24/01/2010

Aceito em 04/10/2010 
